\title{
Downregulation of Mitofusin 2 in Placenta Is Related to Preeclampsia
}

\author{
Jun Yu, ${ }^{1}$ Xijiao Guo, ${ }^{1}$ Ruibao Chen, ${ }^{2}$ and Ling Feng ${ }^{1}$ \\ ${ }^{1}$ Department of Obstetrics and Gynecology, Tongji Hospital, Tongji Medical College, Huazhong University of Science and Technology, \\ Wuhan 430030, China \\ ${ }^{2}$ Department of Urology, Tongji Hospital, Tongji Medical College, Huazhong University of Science and Technology, \\ Wuhan 430030, China
}

Correspondence should be addressed to Jun Yu; yjtj2010@163.com and Ling Feng; lfengtjmu@163.com

Received 12 September 2015; Revised 1 December 2015; Accepted 2 December 2015

Academic Editor: Sung-Hoon Kim

Copyright ( 2016 Jun Yu et al. This is an open access article distributed under the Creative Commons Attribution License, which permits unrestricted use, distribution, and reproduction in any medium, provided the original work is properly cited.

Background. Mitofusin 2 (Mfn2) is a novel mitochondrial protein that is implicated in cellular proliferation and metabolism; however, the role of Mfn2 in preeclampsia (PE) remains unknown. This study aimed to explore the relationship between Mfn2 and PE. Method. Preeclamptic and normal pregnancies were enrolled in a comparative study. The expression of Mfn2 in placenta was detected by qRT-PCR. And the mitochondrial function was detected by ATP assay. Then TEV-1 cells were cultured in hypoxic conditions. mRNA and protein expressions of Mfn2 were detected by qRT-PCR and western blot separately. Cells' viability was detected by MTT. And the mitochondrial function was detected by ATP and mitochondrial membrane potential (MMP) assay. We further knocked down the Mfn2 gene in TEV-1 cells and evaluated the cells' viability. Results. Mfn2 and ATP expressions were significantly decreased in preeclamptic placentae compared to normal placentae. Mfn2 expression level and the viability of TEV-1 cells were reduced during hypoxic conditions. TEV-1 cells' viability, ATP, and MMP levels were also significantly decreased after knockdown of the Mfn2 gene. Conclusions. These results suggest that defects in Mfn2 could cause mitochondrial dysfunction and decrease trophoblastic cells' viability. Therefore, Mfn2 may be functionally involved in the pathogenesis of PE.

\section{Introduction}

$\mathrm{PE}$ is one of the leading causes of maternal and perinatal mortality and morbidity, which affects approximately $5-8 \%$ of all pregnancies, although there is a great degree of variation across regions [1]. The onset of a new episode of hypertension during pregnancy (with persistent diastolic blood pressure > $90 \mathrm{~mm} \mathrm{Hg}$ ) in conjunction with substantial proteinuria $(>0.3 \mathrm{~g} / 24 \mathrm{~h})$ is generally used as the criteria for identifying PE [2].

To date, the etiology and pathogenic mechanisms of PE remain unclear. Increasing evidence suggests that mitochondrial defects play an important role in the initial stages of the pathogenic mechanisms leading to PE $[3,4]$. Thus, some researchers have hypothesized that mitochondrial defects may cause the impairment of trophoblasts that leads to the severe placental disorder observed in PE [3-5]. This hypothesis provides potential new preventative strategies for PE. Evidence is accumulating that mitochondrial dysfunction is responsible for oxygen-sensitive accumulation and degradation, and impaired mitochondrial function is associated with PE syndrome [6,7]. A series of studies have also identified alterations of mitochondrial proteins in the placentae of PE patients [3].

Mfn2 is a protein of the outer mitochondrial membrane that promotes membrane fusion and is involved in the maintenance of the mitochondrial network and bioenergetics. Mfn2 has a potential role in regulating cell proliferation and oxidative metabolism in many cell types [8]. Recently, Mfn2 has been reported to be an important biomarker and therapeutic target molecule for cardiovascular diseases such as hypertension $[9,10]$. However, the functions of Mfn2 in the pathophysiology of PE remain undiscovered. This study was designed to investigate the correlation between Mfn2 and PE. 
TABLE 1: Perinatal characteristics of both study groups.

\begin{tabular}{lccc}
\hline Characteristic & $\begin{array}{c}\text { Control group } \\
(n=16)\end{array}$ & $\begin{array}{c}\text { PE group } \\
(n=16)\end{array}$ & $p$ \\
\hline Weeks of gestation (w) & $36.7 \pm 1.96$ & $36.4 \pm 2.26$ & NS \\
Apgar (1 min) & $7.9 \pm 0.34$ & $7.6 \pm 0.50$ & NS \\
Apgar (5 min) & $8.9 \pm 0.25$ & $8.5 \pm 1.03$ & NS \\
Weight (g) & $2923.9 \pm 608.20$ & $2470.0 \pm 593.96$ & NS \\
Gravida & $2.2 \pm 1.5$ & $1.8 \pm 0.98$ & NS \\
Age (years) & $26.7 \pm 3.91$ & $29.2 \pm 4.78$ & NS \\
BMI & $22.2 \pm 9.36$ & $28.5 \pm 3.62$ & $<0.05$ \\
Blood pressure & & & \\
$\quad$ Systolic & $102.2 \pm 11.27$ & $148.2 \pm 17.28$ & $<0.05$ \\
$\quad$ Diastolic & $72.4 \pm 8.95$ & $114.0 \pm 10.73$ & $<0.05$ \\
\hline
\end{tabular}

NS = not significant.

\section{Materials and Methods}

2.1. Patient Characteristics and Placenta Sampling. This study was approved by the Ethical Committee of Tongji Hospital, Tongji Medical College, Huazhong University of Science and Technology, China. All participants were informed that their placental tissue would be collected and that both their and their newborn's medical information pieces would be used once they were enrolled into this study. Their written consent was obtained before the initiation of the investigation. A case-control, cross-sectional, and comparative study was undertaken. Pregnant women with PE and pregnant women with normal pregnancies were included. All patients were consecutively admitted to each group. Inclusion criteria for both groups were uncomplicated singleton pregnancy, patient age between 20 and 35 years, and gestation > 33 weeks. Normal pregnancy was defined as a pregnancy in which the mother had normal blood pressure (less than $140 / 90 \mathrm{~mm} \mathrm{Hg}$ ), the absence of proteinuria, and the absence of medical or obstetrical complications. PE was defined according to the guidelines recommended by the American College of Obstetricians and Gynecologists (ACOG): onset of hypertension during midpregnancy or late pregnancy, with systolic and diastolic blood pressure equal to or higher than $140 / 90 \mathrm{~mm} \mathrm{Hg}$ on at least two occasions that were separated by $6 \mathrm{~h}$, and proteinuria of more than $300 \mathrm{mg}$ in a $24 \mathrm{~h}$ period after 20 weeks of gestation [11]. The clinical characteristics of the patients are summarized in Table 1. All placental tissues were obtained immediately after delivery. The samples were first thoroughly washed with cold phosphate buffered saline (PBS), and then the villous tissues beneath the chorionic and basal plates were quickly dissected, sliced into small pieces (100-500 mg), snap-frozen in liquid nitrogen, and stored in $-80^{\circ} \mathrm{C}$ freezer.

2.2. Cell Culture and Treatments. The immortalized TEV-1 cell line was obtained from normal extravillous trophoblast cells of a healthy first-trimester human placenta, which was a gift from the Chinese University of Hong Kong [12]. TEV1 cells were grown in DMEM/F12 medium (Sigma-Aldrich,
St. Louis, MO, USA) supplemented with $10 \%$ FBS containing $100 \mathrm{U} / \mathrm{mL}$ penicillin and $0.1 \mathrm{mg} / \mathrm{mL}$ streptomycin and incubated at $37^{\circ} \mathrm{C}$ with $5 \% \mathrm{CO}_{2}$. To detect the effect of hypoxia on the expression of Mfn2 in trophoblastic cells, TEV-1 cells were treated with a hypoxic stimulus $\left(\mathrm{CoCl}_{2}\right)$, as previously described [13]. TEV-1 cells were exposed to $300 \mu \mathrm{M} \mathrm{CoCl}_{2}$ for $0,12,24,48$, or $72 \mathrm{~h}$ after being initially grown at $37^{\circ} \mathrm{C}$ for $24 \mathrm{~h}$. Three replicated wells were used for each group. Following incubation, the cells were harvested for qRT-PCR, western blot, and MTT. HIF-1 $\alpha$ level was used to evaluate the $\mathrm{CoCl}_{2}$-induced hypoxia. To explore the effect of Mfn2 on trophoblastic cell viability, TEV-1 cells were transfected with $50 \mathrm{nM}$ of siRNA-Mfn2 or control oligonucleotides. Transfection was performed with Lipofectamine 2000 in OptiMEM (Invitrogen, Carlsbad, CA, USA) according to the manufacturer's instructions. Cell viability was assessed with transfected TEV-1 cells in 96-well plates using an MTT kit. The function of mitochondria was detected by ATP and MMP assay.

2.3. qRT-PCR Determination of Mfn2 Expression in Placental Villous Tissues. Total RNA was extracted using trizol reagent (Invitrogen, Carlsbad, CA, USA) as described previously [14]. The ratio of ultraviolet absorbance at $260 / 280 \mathrm{~nm}$ was determined as 1.8-2.0. RNA quality was detected by agarose gel electrophoresis. Those RNA, with their 28S:18S being approximately $2: 1$, were subjected to reverse transcription using a kit (Toyobo Co., Ltd., Osaka, Japan) under the conditions, $30^{\circ} \mathrm{C}$ for $10 \mathrm{~min}, 42^{\circ} \mathrm{C}$ for $30 \mathrm{~min}, 99^{\circ} \mathrm{C}$ for $5 \mathrm{~min}$, and $4^{\circ} \mathrm{C}$ for $5 \mathrm{~min}$, and then stored at $-20^{\circ} \mathrm{C}$. qPCR was performed with an Mx3000P Real-Time PCR System using SYBR Green PCR mix (Toyobo Co. Ltd., Osaka, Japan) and the primer pairs, $\beta$-actin, $5^{\prime}$-GGGGTGTTGAAGGTCTCAAA-3' (forward), and $5^{\prime}$-AGAAAATCTGGCACCACACC- $3^{\prime}$ (reverse) and Mfn2, $5^{\prime}$-CCCCCTTGTCTTTATGCTGATGTT-3' (forward), and $5^{\prime}$-TTTTGGGAGAGGTGTTGCTTATTTC- $3^{\prime}$ (reverse). The reaction was carried out under the following conditions: $95^{\circ} \mathrm{C}$ for $2 \mathrm{~min}$, followed by 40 cycles of denaturation at $95^{\circ} \mathrm{C}$ for $30 \mathrm{sec}$, annealing at $60^{\circ} \mathrm{C}$ for $30 \mathrm{sec}$, and extension at $72^{\circ} \mathrm{C}$ for $45 \mathrm{sec}$. The comparative threshold cycle (CT) method was used for relative quantification. All samples were performed in triplicate; data were averaged across the mRNA levels of each sample and then normalized to $\beta$-actin.

\subsection{Cell Viability of Trophoblastic Cells in Hypoxic Conditions} or Treated with siRNA. TEV-1 cells were seeded in 96-well plates at a density of $5 \times 10^{4}$ cells per well and cultured with complete medium for initial $24 \mathrm{~h}$ for attachment. Then, cells were changed into fresh medium containing $300 \mu \mathrm{M} \mathrm{CoCl}_{2}$ and cocultured for $0,12,24,48$, or $72 \mathrm{~h}$. At the end of the time period, TEV-1 cell viability was tested by MTT assay as previously described [15]. Briefly, the treated cells were incubated with fresh media containing MTT for $4 \mathrm{~h}$ at $37^{\circ} \mathrm{C}$ in $5 \% \mathrm{CO}_{2}$. Then, the supernatant was discarded, and $100 \mu \mathrm{L}$ DMSO was added to each well. Absorbance at $590 \mathrm{~nm}$ was measured on a multiwell microplate reader. The results are expressed as a percentage of the control. 
2.5. Western Blot Assay of Mfn2 Expression. Cells were lysed in a lysis buffer at $0^{\circ} \mathrm{C}$. The lysate was centrifuged at $16,000 \mathrm{~g}$ for $30 \mathrm{~min}$, the supernatant was recovered and assayed for protein concentration, and a standard reference curve was obtained with the use of bovine serum albumin. Protein extracts ( $40 \mu \mathrm{g}$ per lane) were separated on a 10\% SDS-PAGE gel and transferred onto PVDF membranes. Then, the membranes were subjected to immunoblot analysis as previously described [16]. Primary rabbit polyclonal anti-Mfn2, HIF$1 \alpha$, and $\beta$-actin antibodies (Santa Cruz Biotechnology Inc., Santa Cruz, CA, USA; 1:1000) and secondary anti-rabbit horseradish peroxidase-linked whole antibody (Santa Cruz Biotechnology Inc., Santa Cruz, CA, USA; 1:5000) were used for these experiments. Antigen-antibody reactions were detected and visualized on film by chemiluminescence. $\beta$ actin was reprobed on the same membrane after washing as an internal control. The intensity of the Mfn 2 and HIF- $1 \alpha$ protein bands was quantified by Quantity One software and normalized to the amount of $\beta$-actin protein in the same sample.

2.6. Measurement of ATP. Direct measurements of ATP levels were obtained using an ATP assay kit according to the manufacturer's instructions (Beyotime, China). The kit is based on a luciferase-luciferin reaction assay. For these experiments, placental tissues and trophoblastic cells were split by the lysis reagent and subjected to centrifuge at $12,000 \mathrm{~g}$ for $5 \mathrm{~min} .100 \mu \mathrm{L}$ of each supernatant was mixed with $100 \mu \mathrm{L}$ ATP working dilution. Luminance was measured using a monochromator microplate reader. The ATP levels were measured and the results were expressed as percentage of the treated control cells. For statistical analysis, the experiments were repeated three times.

2.7. Determination of MMP. Potential MMP was determined using the dual-emission mitochondrial dye JC-1 as described previously [17]. Cells were seeded in black-welled, 96-well plates, and the plate bases were covered before fluorescent reading. Cells were then incubated with JC-1 at a final concentration of $1 \mathrm{mM}$ at $37^{\circ} \mathrm{C}$ for the last $30 \mathrm{~min}$ of the experiment. Red and green fluorescence were quantified using the fluorescent plate reader. The excitation peak was at $490 \mathrm{~nm}$, with the emission maximum at $530 \mathrm{~nm}$ for the monomer, and the excitation peak and the emission maximum for the aggregate were 530 and $600 \mathrm{~nm}$, respectively. The data were expressed as percentage of the treated control cells. And each experiment was repeated three times.

2.8. Statistical Analysis. All the statistical analysis was performed using SPSS 13.0 statistical software. Data are presented as the mean \pm standard deviation. Nonparametric tests were used for skewed data and Student's $t$-test was used for normally distributed numerical variables. $p<0.05$ was accepted as a statistically significant difference.

\section{Results}

3.1. Case-Control Study. Demographic characteristics from our case-control study are shown in Table 1 . There were

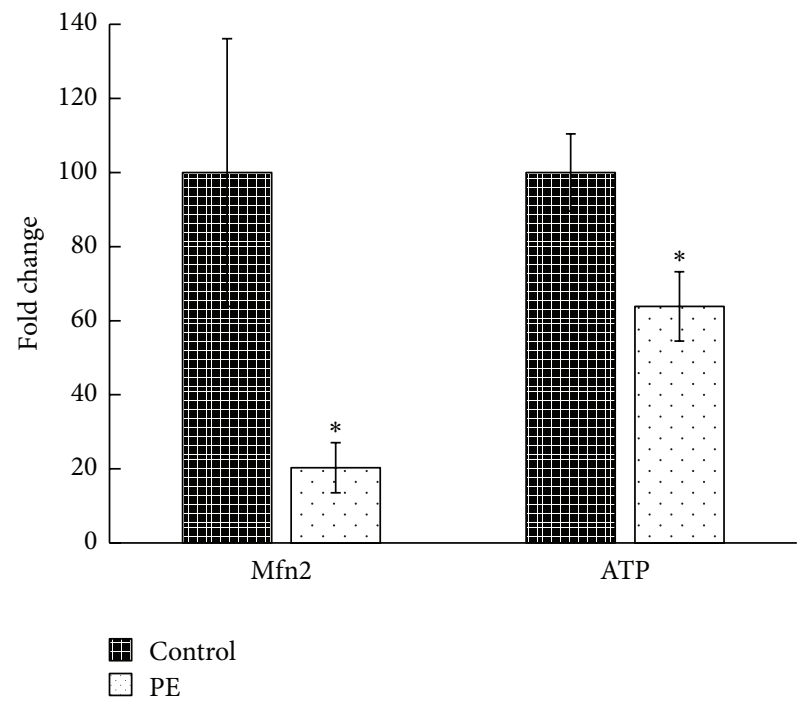

Figure 1: Mfn2 expression and ATP level in villous tissue. The expression of Mfn2 in placental villous tissue from both the control group and the PE group was assessed by qRT-PCR. The ATP level in placental villous tissue was assessed using a luciferase-luciferin reaction assay. The results showed that the Mfn 2 mRNA expression and ATP levels in the villous tissue of the PE group were notably lower than in the control group (data are represented as the mean \pm $\left.\mathrm{SD},{ }^{*} p<0.05\right)$.

no differences in age, weight, or weeks of gestation among the groups. The body mass index (BMI) of the PE group was higher than the control group, and the difference was statistically significant.

3.2. Mfn2 and ATP Levels Were Significantly Decreased in the Placentae from the PE Group. The expression of Mfn2 in placental villous tissue was assessed by qRT-PCR. The ATP level in placental villous tissue was assessed using a luciferase-luciferin reaction assay. The results revealed that the Mfn2 mRNA expression and ATP levels in the villous tissue of the PE group were notably lower than in the control group (Figure 1). These findings suggest that expression of Mfn2 mRNA decreased in the placental villous tissues of patients with PE. And there is mitochondrial dysfunction in the placenta from PE patient.

3.3. Mfn2 Expression in TEV-1 Cells Was Reduced in Hypoxic Conditions. qRT-PCR analysis revealed that the Mfn2 mRNA levels were significantly reduced in TEV-1 cells exposed to $\mathrm{CoCl}_{2}$ as early as $12 \mathrm{~h}$ after exposure and decreased in a time-dependent manner (Figure 2(a)). The western blot results also showed that the Mfn2 expression of trophoblastic cells decreased in a time-dependent manner when exposed to $\mathrm{CoCl}_{2}$, while the HIF-1 $\alpha$ expression level increased (Figure 2(b)).

3.4. TEV-1 Cell Viability Was Reduced in Hypoxic Conditions. The MTT results showed that TEV-1 cell viability significantly decreased when exposed to $\mathrm{CoCl}_{2}$, and this decrease was 

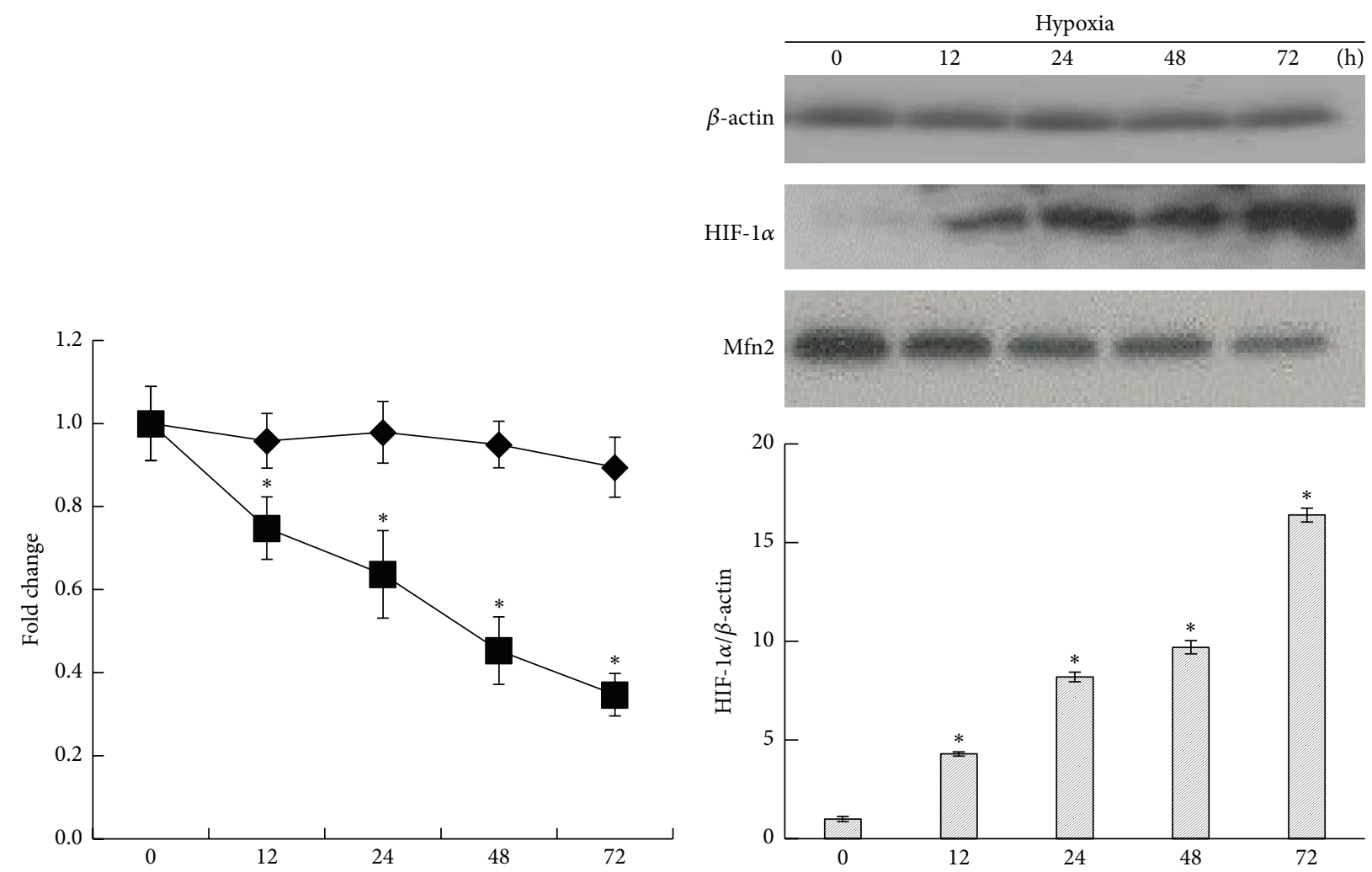

(h)
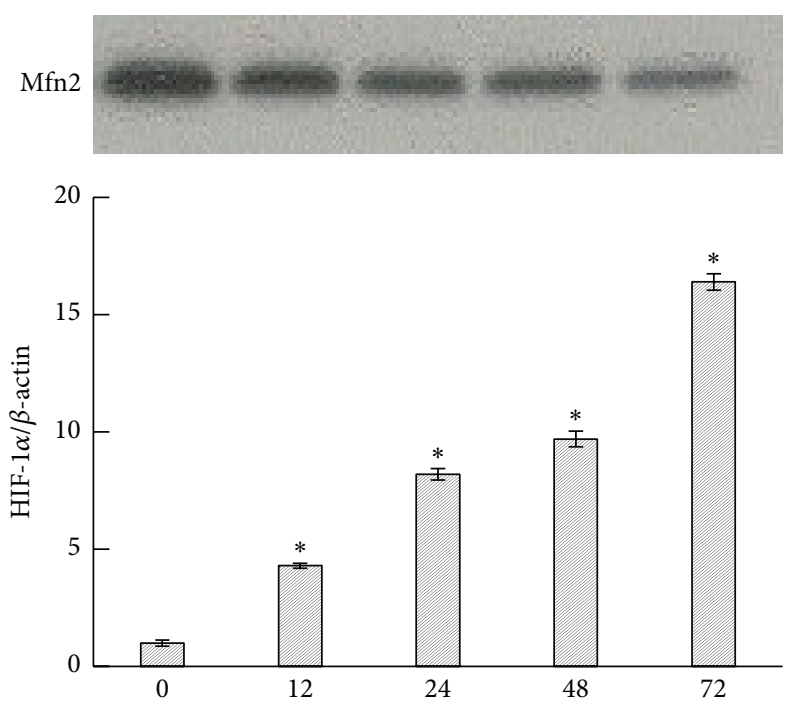

(h)

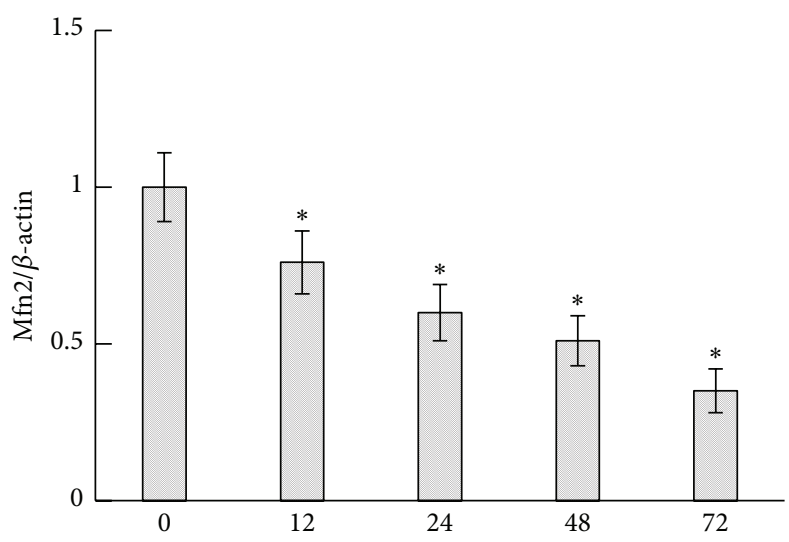

(h)

(a)

(b)

FIgURE 2: Mfn2 expression of trophoblastic cells in hypoxic conditions. TEV-1 cells were treated with $300 \mu \mathrm{M} \mathrm{CoCl} \mathrm{for}_{2}, 12,24,48$, or $72 \mathrm{~h}$. (a) Mfn2 mRNA levels in trophoblastic cells were determined using qRT-PCR. The results showed that $\mathrm{CoCl}_{2}$ treatment reduced Mfn2 mRNA levels in trophoblastic cells as early as $12 \mathrm{~h}$ after treatment, and the reduction was time-dependent $\left({ }^{*} p<0.05\right)$. (b) The western blot results also showed that the Mfn2 protein level of trophoblastic cells decreased in a time-dependent manner when exposed to $\mathrm{CoCl}_{2}$, while the HIF-1 $\alpha$ expression level increased $\left({ }^{*} p<0.05\right)$. All data are presented as the mean \pm SD of three independent experiments.

observed as early as $24 \mathrm{~h}$ after treatment (Figure 3 ). TEV1 cell viability also decreased in a time-dependent manner when exposed to $\mathrm{CoCl}_{2}$. The trend in TEV-1 cell viability in the hypoxic environment was similar to the trend in Mfn2 expression level, while the decrease of TEV-1 cell viability followed the decrease of Mfn2 expression level.
3.5. TEV-1 Cell Viability Decreased after Mfn2 Knockdown. To explore whether Mfn2 is integral to the survival of TEV-1 cells in culture, TEV-1 cells were treated with siRNA-Mfn2 or control oligonucleotides and allowed to recover in full growth media for approximately 48 hours; subsequently, cells were incubated in low-mitogen media overnight. Knockdown of 


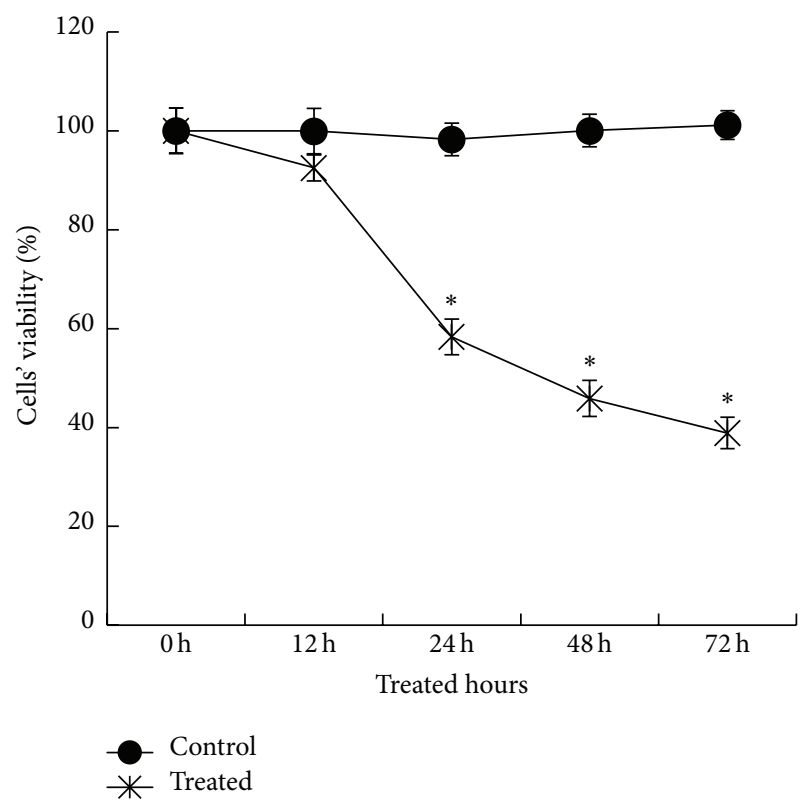

FIGURE 3: The viability of trophoblastic cells in hypoxic conditions. TEV-1 cells were treated with $300 \mu \mathrm{M} \mathrm{CoCl}_{2}$ for $0,12,24,48$, or $72 \mathrm{~h}$. Then, TEV-1 cell viability was determined by the MTT method. Data are presented as the mean \pm SD of three independent experiments. The results showed that TEV-1 cell viability significantly decreased when cells were exposed to $\mathrm{CoCl}_{2}$, and the reduction was observed as early as $24 \mathrm{~h}$ after treatment. In addition, the reduction was timedependent $\left({ }^{*} p<0.05\right)$.

Mfn2 led to $81.33 \pm 3.61 \%$ reduction of Mfn2 protein (Figure 4(a)). Cellular viability was assessed via an MTT assay. Upon serum deprivation, a significant loss of viability was observed with the attenuation of Mfn2 (Figure 4(b)).

3.6. ATP Level and MMP in TEV-1 Cell Decreased after Mfn2 Knockdown. To explore mitochondrial function after Mfn2 knockdown, ATP level and MMP level were detected in TEV1 cells treated with siRNA-Mfn2 or control. The results show that knockdown of Mfn2 led to significant reduction of both ATP level and MMP in trophoblastic cells (Figure 5).

\section{Discussion}

Placental dysfunction is the most common pathological change in pregnancy complications, which include PE. Recently, placental mitochondrial dysfunction has drawn the attention of researchers. Placental mitochondria provide most of the energy production in cells, participate in a number of important cellular processes, and are likely to play a central role in placental implantation, growth, and development. Some investigative teams have suggested that mitochondrial dysfunction may contribute to the pathogenesis of PE $[3,18]$. However, there has been little research investigating the functional status of placental mitochondria in PE.

Mfn2 has been reported to be correlated with the pathological changes of some diseases related to mitochondrial signaling. In the present study, we found that the expression of Mfn2 in the placentae of the PE group was significantly decreased compared to the control group, which suggests that Mfn2 may be involved in the pathology of PE. We also measured the ATP, which represented key pathophysiological conditions in mitochondria [19]. Our results show that the placentae from PE patients possess a lower ATP level. These results confirmed the mitochondrial dysfunction in PE. PE is a pregnancy-specific disease characterized by the novel onset of hypertension and a series of other systematic disorders caused by renal injury (such as proteinuria and edema). Recent research demonstrates that Mfn 2 could be an important biomarker and therapeutic target molecule for cardiovascular diseases such as hypertension. The expression of Mfn2 was markedly downregulated in vascular smooth muscle cells in spontaneously hypertensive rats [9]. Decreased expression of Mfn2 has also been found to contribute to mitochondrial fragmentation and a proliferation-cell death imbalance in human and experimental pulmonary arterial hypertension [10]. Other authors have also reported that Mfn2 deficiency exacerbates renal epithelial cell injury by promoting mitochondrial outer membrane injury and cell death [20]. Together with our results, these findings indicate that Mfn2 may be a potential biomarker and therapeutic target molecule for PE.

We further explored how Mfn2 expression in trophoblastic cells varies under hypoxic conditions. The results showed that the expression of Mfn2 mRNA in trophoblastic cells decreased in a time-dependent manner in the presence of a hypoxic stimulus, and the expression of Mfn2 was negatively correlated with HIF-1 $\alpha$. We also found that hypoxia significantly reduced TEV-1 cell viability. Both the Mfn2 expression level and the viability of TEV-1 cells were decreased in hypoxic conditions, although the decrease in Mfn2 expression level was observed prior to the decrease in TEV-1 cell viability. Previous studies have demonstrated that chorionic hypoxia can induce the trophoblast dysfunction and placental insufficiency syndromes of PE [21]. Our research shows negative relationship between Mfn2 and HIF- $1 \alpha$. These results combined the hypoxia-induced preeclampsia theory and mitochondrial dysfunction in PE. However, Mfn2 plays an important role in the pathogenesis of PE. Mfn2 is an important mitochondrial protein in the maintenance of mitochondrial bioenergetics and morphology. Recently, Mfn2 has been reported to have a potential role in regulating cell proliferation in many cell types [22, 23]. Mfn2 was found to have an important role in the activation of mitochondrial apoptosis pathways in cells; thus, the degradation of Mfn2 would lead to mitochondrial fragmentation and enhanced cell death [24]. A previous study also suggests that low Mfn2 expression is associated with apoptosis in the placental villi, which is consistent with our results [25]. Thus, our research could partially explain the decreased proliferation of trophoblasts, strengthening the hypothesis that Mfn2 plays a key role in trophoblast cell viability and acts as part of the molecular mechanisms underlying mitochondrial dysfunction in PE.

To confirm that Mfn2 is important for the survival of TEV-1 cells, we used siRNA to knock down the expression 

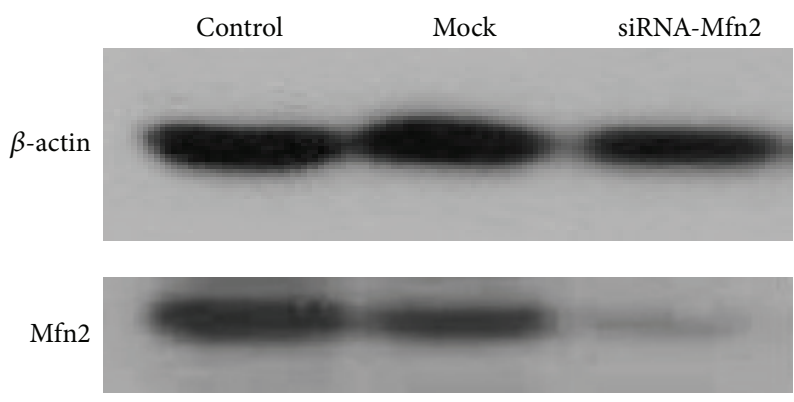

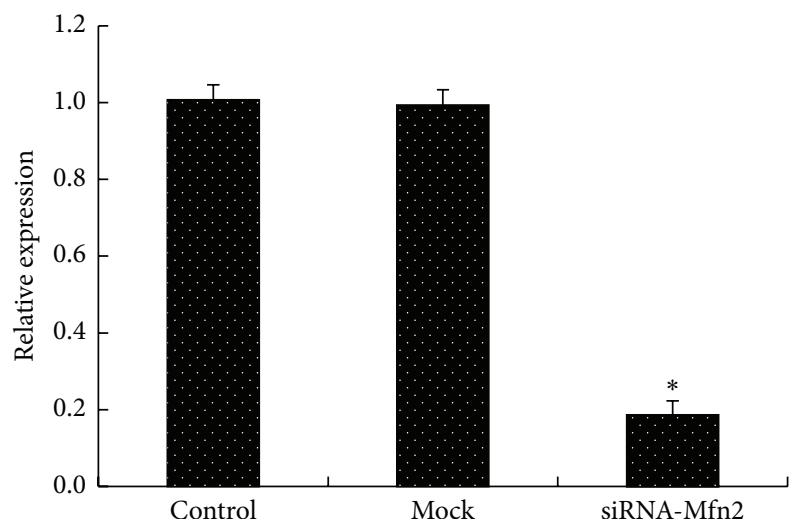

(a)

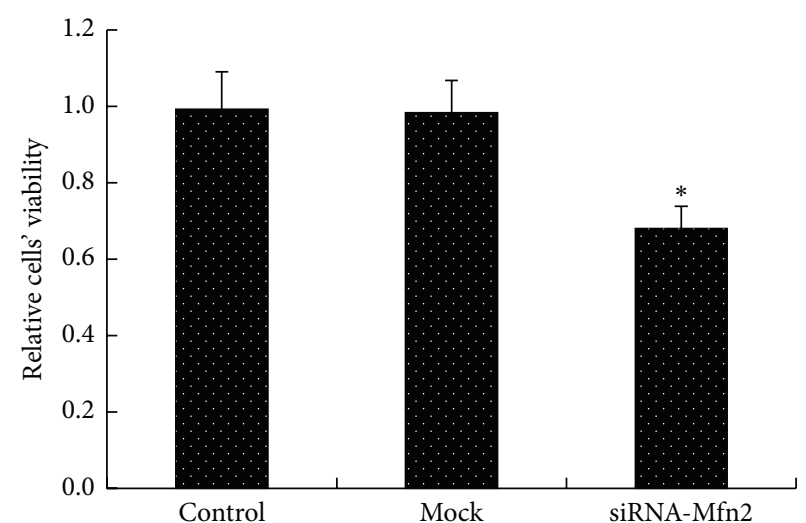

(b)

Figure 4: The viability of trophoblastic cells after Mfn2 knockdown. TEV-1 cells were transfected with $50 \mathrm{nM}$ of siRNA-Mfn2 or control oligonucleotides. (a) The protein expression of Mfn2 in cells from the control group, mock group, and siRNA-Mfn2 group was detected by western blot. The results showed that the Mfn2 knockdown led to $81.33 \pm 3.61 \%$ reduction of Mfn2 protein $\left({ }^{*} p<0.05\right)$. (b) Trophoblastic cells from the control group, mock group, and siRNA-Mfn2 group were allowed to recover in full growth media for approximately $48 \mathrm{~h}$ before being incubated in low-mitogen media overnight. Then, cellular viability was assessed via an MTT assay. A significant loss of viability was observed with attenuation of Mfn2 (data are represented as the mean $\pm \mathrm{SD},{ }^{*} p<0.05$ ).

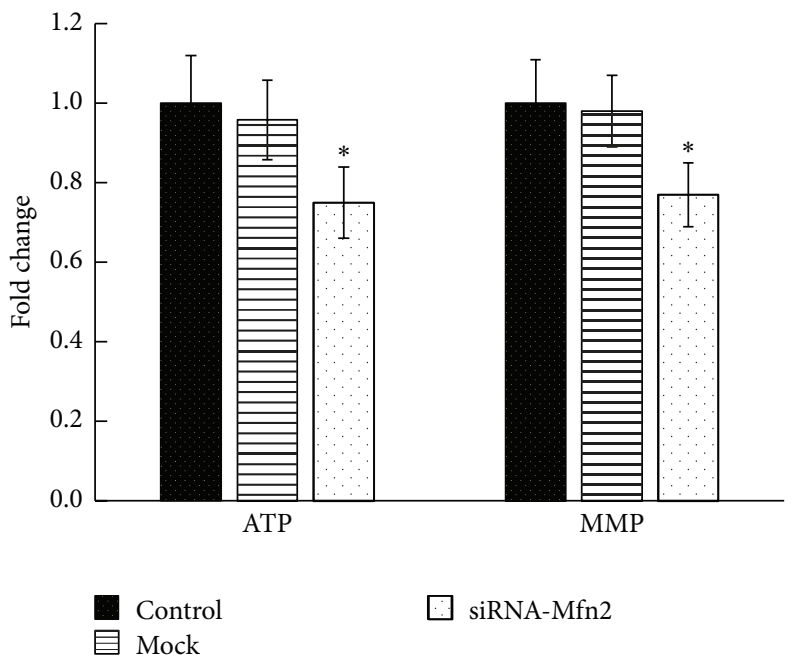

FIGURE 5: The ATP and MMP levels of trophoblastic cells after Mfn2 knockdown. The ATP and MMP were quantified based on fluorescence intensities. ATP level and MMP were both significantly decreased in siRNA-Mfn2 group, compared to the control group $\left({ }^{*} p<0.05\right)$. of Mfn2. To assess mitochondrial injury, we measured the MMP and ATP level. Our results indicated that Mfn2 knockdown leads to significant decrease of MMP and ATP levels. When Mfn2 expression was knocked down, cellular viability was also notably decreased. These results further indicated that the reduction of Mfn2 expression could reduce the proliferation of trophoblasts. Lugus et al. have reported that Mfn2 ablation diminishes endothelial cell viability and disrupts endothelial cell tube formation [26]. These results are consistent with our findings. Trophoblast cells exhibit high proliferation rates and play a crucial role in vascular remodeling of the placenta [27]. Any factors that lead to reduced trophoblast cell proliferation may result in poor vascular remodeling of the placenta, which is a key feature of PE.

Increasing evidence indicates that Mfn2 is strong regulator of metabolism. Mfn2 deficiency is associated with enhanced hydrogen peroxide concentrations, altered reactive oxygen species regulation, insulin resistance, and endoplasmic reticulum stress [28]. In this study, we also found that BMIs in the PE group were higher than in the control group. This result is consistent with previous reports [29]. However, 
the mechanism by which Mfn2 could regulate metabolism in the placenta needs further study. The results presented here provide evidence that Mfn2 may play a role in the multifactorial pathogenic mechanisms of PE.

Our research revealed that the level of Mfn2 in the PE group was notably lower than the level in the normal pregnancy group. This research also demonstrated that hypoxia decreased the expression of Mfn2 in trophoblastic cells, as well as cells' viability. And Mfn2 knockdown led to diminishing of trophoblastic cells' viability. Therefore, Mfn2 may be functionally involved in the pathogenesis of PE.

\section{Conflict of Interests}

The authors declared that there is no conflict of financial interests related to this study.

\section{Acknowledgments}

The authors thank the staff of Tongji Hospital and the patients for donating their placentae. This work was supported by grant from National Natural Science Foundation of China (no. J1103514).

\section{References}

[1] WHO Guidelines Approved by the Guidelines Review Committee, WHO Recommendations for Prevention and Treatment of Pre-Eclampsia and Eclampsia, World Health Organization, Geneva, Switzerland, 2011.

[2] E. Abalos, C. Cuesta, A. L. Grosso, D. Chou, and L. Say, "Global and regional estimates of preeclampsia and eclampsia: a systematic review," European Journal of Obstetrics Gynecology and Reproductive Biology, vol. 170, no. 1, pp. 1-7, 2013.

[3] Z. Shi, W. Long, C. Zhao, X. Guo, R. Shen, and H. Ding, "Comparative proteomics analysis suggests that placental mitochondria are involved in the development of pre-eclampsia," PLoS ONE, vol. 8, no. 5, Article ID e64351, 2013.

[4] S. Muralimanoharan, A. Maloyan, J. Mele, C. Guo, L. G. Myatt, and L. Myatt, "MIR-210 modulates mitochondrial respiration in placenta with preeclampsia," Placenta, vol. 33, no. 10, pp. 816823, 2012.

[5] M. Widschwendter, H. Schröcksnadel, and M. G. Mörtl, "Preeclampsia: a disorder of placental mitochondria?" Molecular Medicine Today, vol. 4, no. 7, pp. 286-291, 1998.

[6] H. Teng, B. Wu, K. Zhao, G. Yang, L. Wu, and R. Wang, "Oxygen-sensitive mitochondrial accumulation of cystathionine $\beta$-synthase mediated by Lon protease," Proceedings of the National Academy of Sciences of the United States of America, vol. 110, no. 31, pp. 12679-12684, 2013.

[7] S. Illsinger, N. Janzen, S. Sander et al., "Preeclampsia and HELLP syndrome: impaired mitochondrial function in umbilical endothelial cells," Reproductive Sciences, vol. 17, no. 3, pp. 219-226, 2010.

[8] W. Wang, J. Lu, F. Zhu et al., "Pro-apoptotic and antiproliferative effects of mitofusin-2 via Bax signaling in hepatocellular carcinoma cells," Medical Oncology, vol. 29, no. 1, pp. 70-76, 2012.

[9] L. Fang, X.-L. Moore, X.-M. Gao, A. M. Dart, Y. L. Lim, and X.J. Du, "Down-regulation of mitofusin-2 expression in cardiac hypertrophy in vitro and in vivo," Life Sciences, vol. 80, no. 23, pp. 2154-2160, 2007.

[10] J. J. Ryan, G. Marsboom, Y.-H. Fang et al., "PGCl $\alpha$-mediated mitofusin-2 deficiency in female rats and humans with pulmonary arterial hypertension," American Journal of Respiratory and Critical Care Medicine, vol. 187, no. 8, pp. 865-878, 2013.

[11] ACOG Committee on Practice Bulletins-Obstetrics, "ACOG practice bulletin. Diagnosis and management of preeclampsia and eclampsia," Obstetrics and Gynecology, vol. 99, no. 1, pp. 159167, 2002.

[12] H. C. Feng, M. Y. Choy, W. Deng et al., "Establishment and characterization of a human first-trimester extravillous trophoblast cell line (TEV-1)," Journal of the Society for Gynecologic Investigation, vol. 12, no. 4, pp. e21-e32, 2005.

[13] M. Andrews and M. Arredondo, "Hepatic and adipocyte cells respond differentially to iron overload, hypoxic and inflammatory challenge," BioMetals, vol. 25, no. 4, pp. 749-759, 2012.

[14] J. Yu, Y. Zhou, J. Gui, A.-Z. Li, X.-L. Su, and L. Feng, "Assessment of the number and function of macrophages in the placenta of gestational diabetes mellitus patients," Journal of Huazhong University of Science and Technology [Medical Sciences], vol. 33, no. 5, pp. 725-729, 2013.

[15] J. Ho, Y. Du, O. G.-W. Wong, M. K. Y. Siu, K. K. L. Chan, and A. N. Y. Cheung, "Downregulation of the gli transcription factors regulator kif7 facilitates cell survival and migration of choriocarcinoma cells," PLoS ONE, vol. 9, no. 9, Article ID e108248, 2014.

[16] A. N. Sharp, A. E. P. Heazell, D. Baczyk et al., "Preeclampsia is associated with alterations in the p53-pathway in villous trophoblast," PLoS ONE, vol. 9, no. 1, Article ID e87621, 2014.

[17] Z. Wang, X. Tang, Y. Li et al., "20-Hydroxyeicosatetraenoic acid inhibits the apoptotic responses in pulmonary artery smooth muscle cells," European Journal of Pharmacology, vol. 588, no. 1, pp. 9-17, 2008.

[18] D. Ding, N. M. Scott, E. E. Thompson et al., "Increased proteincoding mutations in the mitochondrial genome of African American women with preeclampsia," Reproductive Sciences, vol. 19, no. 12, pp. 1343-1351, 2012.

[19] C. Peng, W. Rao, L. Zhang et al., "Mitofusin 2 ameliorates hypoxia-induced apoptosis via mitochondrial function and signaling pathways," The International Journal of Biochemistry \& Cell Biology, vol. 69, pp. 29-40, 2015.

[20] J. M. Gall, Z. Wang, M. Liesa et al., "Role of mitofusin 2 in the renal stress response," PLoS ONE, vol. 7, no. 1, Article ID e31074, 2012.

[21] S. Y. Jeon, H.-J. Lee, K.-H. Na et al., "Hypoxia-induced downregulation of XIAP in trophoblasts mediates apoptosis via interaction with IMUP-2: implications for placental development during pre-eclampsia," Journal of Cellular Biochemistry, vol. 114, no. 1, pp. 89-98, 2013.

[22] D. Zhang, C. Ma, S. Li et al., "Effect of Mitofusin 2 on smooth muscle cells proliferation in hypoxic pulmonary hypertension," Microvascular Research, vol. 84, no. 3, pp. 286-296, 2012.

[23] W. Wang, Q. Sun, Z. Wu et al., "Mitochondrial dysfunctionrelated genes in hepatocellular carcinoma," Frontiers in Bioscience, vol. 18, no. 3, pp. 1141-1149, 2013.

[24] G. P. Leboucher, Y. C. Tsai, M. Yang et al., "Stress-induced phosphorylation and proteasomal degradation of mitofusin 2 facilitates mitochondrial fragmentation and apoptosis," Molecular Cell, vol. 47, no. 4, pp. 547-557, 2012. 
[25] W. Pang, Y. Zhang, N. Zhao, S. S. Darwiche, X. Fu, and W. Xiang, "Low expression of Mfn2 is associated with mitochondrial damage and apoptosis in the placental villi of early unexplained miscarriage," Placenta, vol. 34, no. 7, pp. 613-618, 2013.

[26] J. J. Lugus, G. A. Ngoh, M. M. Bachschmid, and K. Walsh, "Mitofusins are required for angiogenic function and modulate different signaling pathways in cultured endothelial cells," Journal of Molecular and Cellular Cardiology, vol. 51, no. 6, pp. 885-893, 2011.

[27] K. A. Pennington, J. M. Schlitt, D. L. Jackson, L. C. Schulz, and D. J. Schust, "Preeclampsia: multiple approaches for a multifactorial disease," Disease Models and Mechanisms, vol. 5, no. 1, pp. 9-18, 2012.

[28] D. Sebastián, M. I. Hernández-Alvarez, J. Segalés et al., "Mitofusin 2 (Mfn2) links mitochondrial and endoplasmic reticulum function with insulin signaling and is essential for normal glucose homeostasis," Proceedings of the National Academy of Sciences of the United States of America, vol. 109, no. 14, pp. 55235528, 2012.

[29] A. Aksornphusitaphong and V. Phupong, "Risk factors of early and late onset pre-eclampsia," Journal of Obstetrics and Gynaecology Research, vol. 39, no. 3, pp. 627-631, 2013. 


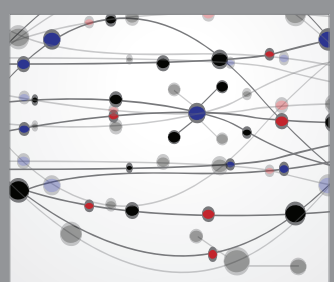

The Scientific World Journal
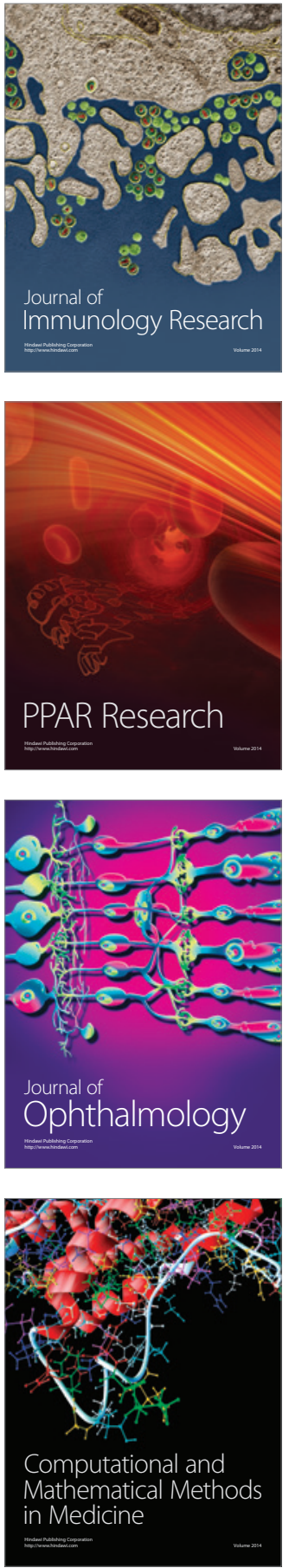

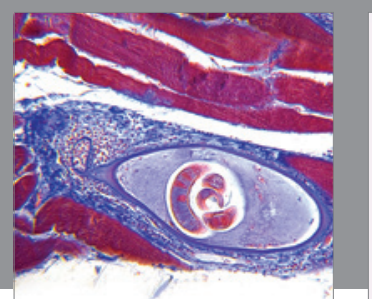

Gastroenterology Research and Practice

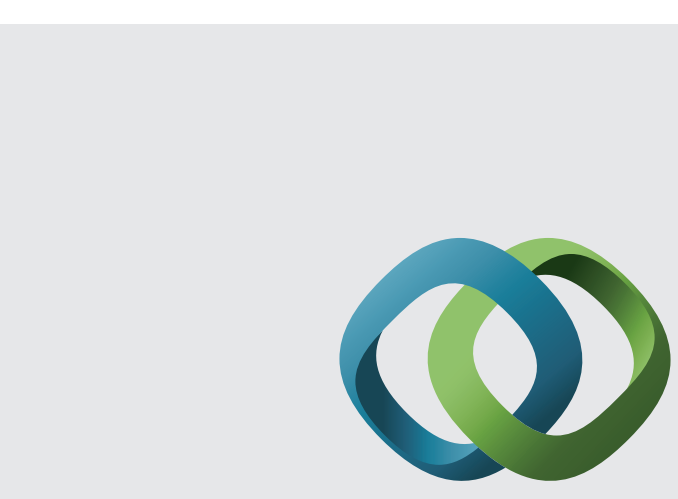

\section{Hindawi}

Submit your manuscripts at

http://www.hindawi.com
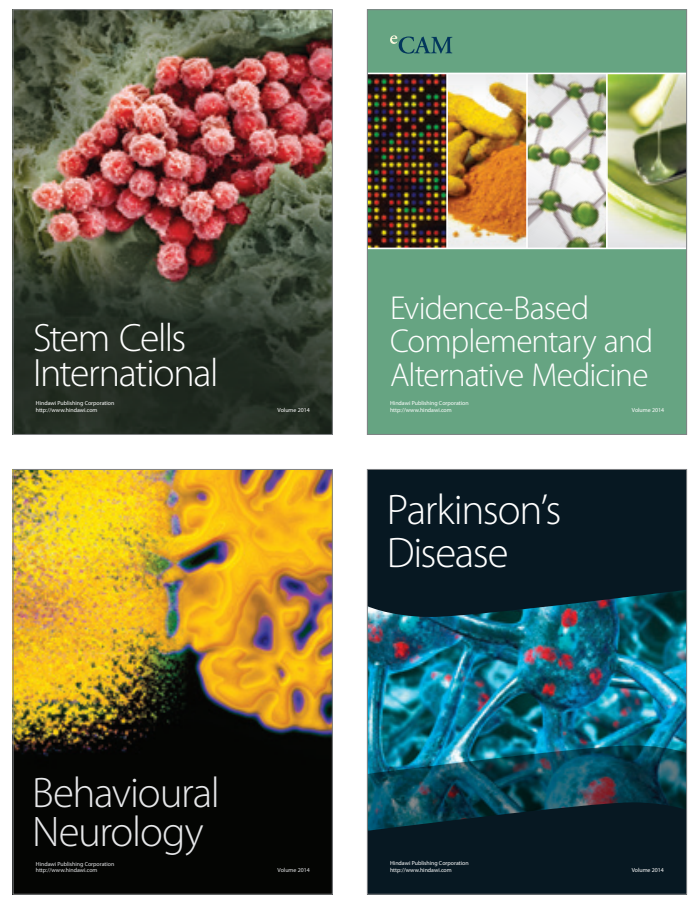
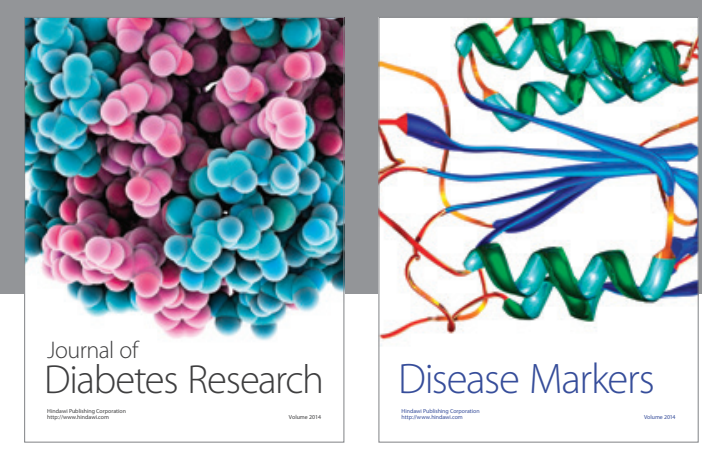

Disease Markers
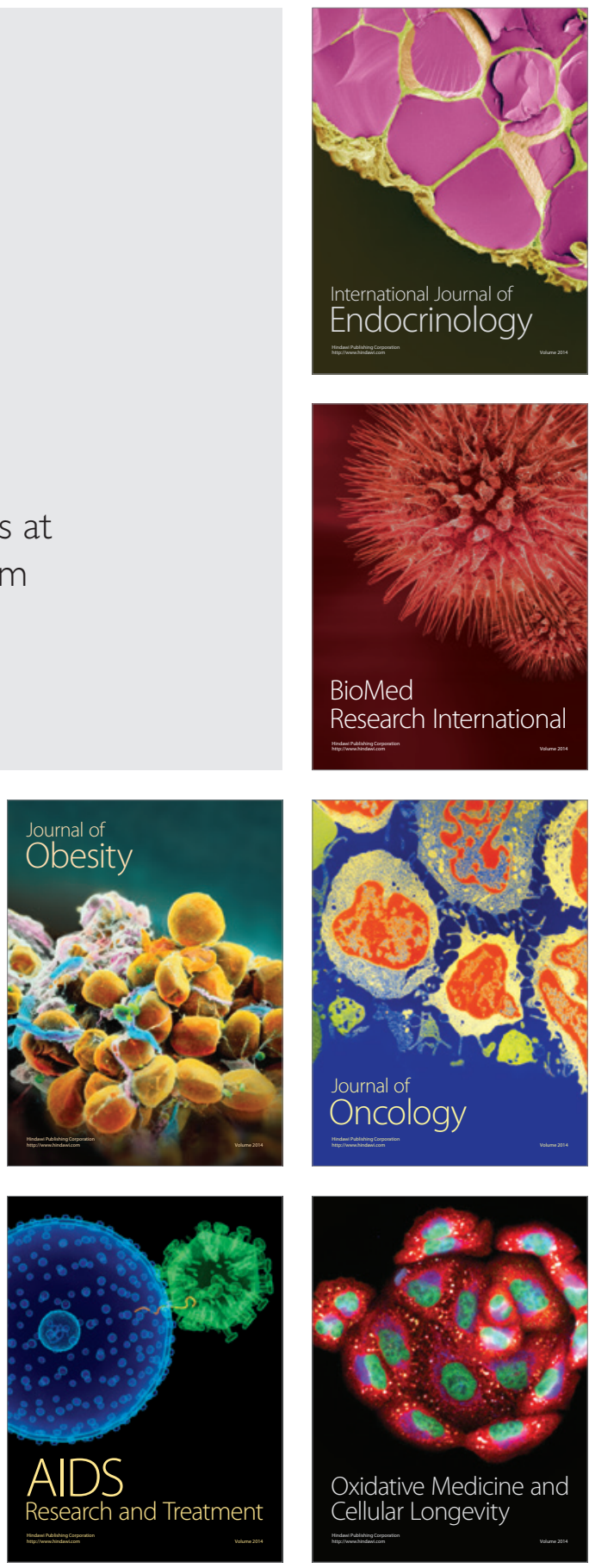